\title{
Enhanced Expression of Fibroblast Growth Factors and Receptor FGFR-1 during Vascular Remodeling in Chronic Obstructive Pulmonary Disease
}

\author{
Andor R. Kranenburg, Willem I. de Boer, J. Han J.M. van Krieken, Wolter J. Mooi, Jane E. Walters, \\ Pramod R. Saxena, Peter J. Sterk, and Hari S. Sharma \\ Department of Pharmacology, Erasmus University Medical Center, Rotterdam; Departments of Pulmonology and Pathology, \\ Leiden University Medical Center, Leiden; Department of Pathology, Netherlands Cancer Institute, Amsterdam, The \\ Netherlands; and School of Biological Molecular Sciences, Oxford Brookes University, Oxford, United Kingdom
}

\begin{abstract}
Important characteristics of chronic obstructive pulmonary disease (COPD) include airway and vascular remodeling, the molecular mechanisms of which are poorly understood. We assessed the role of fibroblast growth factors (FGF) in pulmonary vascular remodeling by examining the expression pattern of FGF-1, FGF-2, and the FGF receptor (FGFR-1) in peripheral area of lung tissues from patients with COPD $\left(\mathrm{FEV}_{1} \leqslant 75 \% ; n=15\right)$ and without $\mathrm{COPD}\left(\mathrm{FEV}_{1} \geqslant 85 \% ; n=13\right)$. Immunohistochemical staining results were evaluated by digital video image analysis as well as by manual scoring. FGF-1 and FGFR-1 were detected in vascular smooth muscle (VSM), airway smooth muscle, and airway epithelial cells. FGF-2 was localized in the cytoplasm of airway epithelium and in the nuclei of airway smooth muscle, VSM, and endothelial cells. In COPD cases, an unequivocal increase in FGF-2 expression was observed in VSM (3-fold, $P=$ 0.001 ) and endothelium (2-fold, $P=0.007$ ) of small pulmonary vessels with a luminal diameter under $200 \mu \mathrm{m}$. In addition, FGFR-1 levels were elevated in the intima (1.5-fold, $P=0.05)$. VSM cells of large $(>200 \mu \mathrm{m})$ pulmonary vessels showed increased staining for FGF-1 (1.6-fold, $P<0.03$ ) and FGFR-1 (1.4-fold, $P<0.04$ ) in COPD. Pulmonary vascular remodeling, assessed as the ratio of $\alpha$-smooth muscle actin staining and vascular wall area with the lumen diameter, was increased in large vessels of patients with COPD $(P=0.007)$ and was inversely correlated with $\mathrm{FEV}_{1}$ values $(P<0.007)$. Our results suggest an autocrine role of the FGF-FGFR-1 system in the pathogenesis of COPD-associated vascular remodeling.
\end{abstract}

Chronic obstructive pulmonary disease (COPD) is a global health problem with increasing morbidity and mortality (1). One of the major causal factors is tobacco smoking (2). However, only ten percent of all smokers develop symptomatic COPD. The causes of this variability in response of the airways and lung parenchyma to tobacco smoke exposure have remained largely unclear. One of the key pathologic features of COPD is thickening of airway walls as a result of inflammation, hyperplasia of airway smooth muscle cells

(Received in original form December 15, 2000 and in revised form May 6, 2002) Address correspondence to: Hari S. Sharma, M.Phil., Ph.D., Institute of Pharmacology, Erasmus University Medical Center, Dr. Molewaterplein 50, 3015 GE Rotterdam, The Netherlands. E-mail: sharma@farma.fgg.eur.nl

Abbreviations: chronic obstructive pulmonary disease, COPD; forced expiratory volume in one second, $\mathrm{FEV}_{1}$; fibroblast growth factors, FGF; fibroblast growth factor receptor, FGFR; forced vital capacity, FVC; inside diameter, ID; carbon monoxide diffusion, $\mathrm{K}_{\mathrm{co}}$; platelet-derived growth factor, PDGF; $\alpha$-smooth muscle actin, $\alpha$-SMA; vascular smooth muscle, VSM; vascular wall, VW.

Am. J. Respir. Cell Mol. Biol. Vol. 27, pp. 517-525, 2002 DOI: $10.1165 / \mathrm{rcmb} .4474$

Internet address: www.atsjournals.org and fibroblasts, and increased deposition of extracellular matrix (3). In addition, advanced COPD leads to pathologic changes in the pulmonary circulation $(4,5)$. At least part of this is probably the result of alveolar hypoxia, which is well known to cause pulmonary vasoconstriction and, if the hypoxic stimulus persists, pulmonary vascular remodeling, of which increased muscularization of small arterial branches is the most striking feature (6). With sustained vasoconstriction of pulmonary arteries, arterioles, and veins, the medial vascular smooth muscle (VSM) extends distally to vessels normally devoid of smooth muscle (6). Intimal thickening due to fibrosis and emergence of smooth muscle cells within the intima of small pulmonary arterial branches has also been reported (5). Finally, loss of the pulmonary vascular bed by emphysema has been suggested to lead to the formation of new vessels (6). Thus, several phenomena acting in concert in COPD result in pulmonary vascular remodeling. Yet little is known about the molecular mechanisms underlying these processes in the context of COPD.

A variety of growth factors and cytokines released from various sites of airway and vascular walls (VW) have the potential to contribute to the pathogenesis of vascular remodeling in COPD. In view of their important role in chronic inflammation, fibrosis, and repair of various tissues, including the lung (9), fibroblast growth factors (FGFs) may well play a pivotal role in airway and VW remodeling $(7,8)$. FGFs exert their biologic effects via binding to four high-affinity FGF transmembrane tyrosine-kinase receptors (FGFR) (9). Distinct FGF subtypes bind with different affinity to the various FGFR. Alternative splicing and regulated protein trafficking further modulate the intracellular events and resultant response initiated by FGF ligandreceptor interaction (9). In the lung as well as in the vascular system, FGFs have been implicated in several pathologic conditions. FGF-1 and FGFR-1 were shown to be upregulated during the development of lung fibrosis (10). FGF-2 and platelet-derived growth factor (PDGF) have been implicated in the pathogenesis of obliterative bronchiolitis after transplantation (11). Moreover, vascular remodeling in response to increased blood pressure is associated with elevated levels of basic FGF $(12,13)$.

To investigate whether the FGF-FGFR system might be involved in the pathogenesis of COPD, we examined the expression patterns of FGF-1, FGF-2, and FGFR-1 in smokers and exsmokers with or without COPD and correlated the expression with histologic evidence of pulmonary vascular remodeling. 


\section{Materials and Methods}

\section{Selection of Patients' Specimens}

We examined lung tissue specimens of subjects with or without COPD. Peripheral parts of the lung tissue from current and exsmokers who underwent lobectomy or pneumonectomy for lung cancer was obtained from the Pathology Laboratories of the Leiden University Medical Center, Leiden, the Netherlands, and the Zuiderziekenhuis, Rotterdam, The Netherlands. Tissue specimens were taken distally from the lung hilus and predominantly consisted of parenchyma, small airways, and vasculature. All lung tissues were inflated by an injection syringe using formalin and fixed for $\sim 24 \mathrm{~h}$ after which the tissues were further dehydrated and embedded in paraffin. These tissues were subsequently processed for immunohistochemical staining. Based on a number of lung function data, patients were assigned to the groups with and without COPD $(14,15)$.

Group with COPD. Fifteen subjects were assigned to the COPD group on the basis of the following parameters: forced expiratory volume in one second $\left(\mathrm{FEV}_{1}\right)<75 \%$ of predicted value (16) before bronchodilation, $\mathrm{FEV}_{1}$ /forced vital capacity $(\mathrm{FVC})$ ratio $<$ $75 \%$, a reversibility in $\mathrm{FEV}_{1} \leqslant 12 \%$ of predicted after $400 \mu \mathrm{g}$ inhaled salbutamol, and a transfer factor for carbon monoxide (diffusion capacity) per liter alveolar volume $\left(\mathrm{K}_{\mathrm{co}}\right) \leqslant 80 \%$ of predicted value.

Group without COPD. Thirteen subjects were assigned to the group without COPD on the basis of the following data; $\mathrm{FEV}_{1}>$ $85 \%$ before bronchodilatation, $\mathrm{FEV}_{1} / \mathrm{FVC}$ ratio $>85 \%$, and reversibility in $\mathrm{FEV}_{1} \leqslant 12 \%$ of predicted after $400 \mu \mathrm{g}$ salbutamol inhalation. To exclude accompanying lung disease leading to a restrictive function disorder, the total lung capacity of each subject included in the study was over $80 \%$ of the predicted values (16).

Clinical data of all patients were examined for possible comorbidity and medication usage. All patients were free of symptoms of upper respiratory tract infection and none received antibiotics perioperatively. None of the patients received glucocorticosteroids in the 3 mo before operation; four patients received oral glucocorticosteroids perioperatively. In addition to the rigorous criteria based on lung function parameters, microscopic exclusion criteria was also applied in the selection of patients for this study. After the selection based on lung function, all the lung tissues were subsequently examined histologically by two experienced lung pathologists using the following exclusion criteria: $(i)$ presence of tumor in the lung tissue specimen; (ii) presence of poststenotic pneumonia in the specimen; (iii) fibrosis of lung tissue; and (iv) obstruction of the main bronchus of the resection specimen by tumor $(14,15)$.

\section{Pulmonary Function Tests}

All pulmonary function tests were performed within the 3 months before surgery. $\mathrm{FEV}_{1}$ and $\mathrm{FVC}$ were measured by spirometry, total lung capacity, and residual volume with the closed circuit helium dilution test and the $\mathrm{K}_{\mathrm{co}}$ using the single breath-holding technique, as described by Quanjer and coworkers (16). Lung function data and other patient characteristics are shown in Table 1.

\section{Immunohistochemistry}

Sections of paraffin-embedded lung tissue were cut at $4 \mu \mathrm{m}$, mounted on Super Frost Plus microscopic slides (Menzel-Gläser, Braunschweig, Germany) and processed for immunohistochemistry. Serial sections were used for immunostaining of FGF-1, FGF-2, and FGFR-1 using human-specific antibodies. The optimal dilutions for all antibodies were identified by examining the intensity
TABLE 1

Subject characteristics and clinical parameters

\begin{tabular}{lcc}
\hline & $\begin{array}{c}\text { Subjects without } \\
\text { COPD }\end{array}$ & $\begin{array}{c}\text { Patients with } \\
\text { COPD }\end{array}$ \\
\hline FEV $_{1}$ & $99 \pm 1.9$ & $53 \pm 3.2^{*}$ \\
dFEV $_{1}, \%$ predicted & $3 \pm 0.6$ & $4 \pm 0.9$ \\
FEV1/FVC, \% & $100 \pm 2.3$ & $58 \pm 5.0^{*}$ \\
TLC & $104 \pm 2.0$ & $108 \pm 8.8$ \\
RV & $115 \pm 5.5$ & $141 \pm 15.4^{*}$ \\
Kco, \% predicted & $94 \pm 2.0$ & $55 \pm 5.4^{*}$ \\
Sex (male/female) & $11 / 2$ & $14 / 1$ \\
Age (yr) & $57 \pm 3.2$ & $59 \pm 5.0$ \\
Smokers/exsmokers/nonsmokers & $9 / 4 / 0$ & $12 / 3 / 0$ \\
Pack-years & $33 \pm 4.7$ & $35 \pm 5.2$ \\
Steroid use (yes/no/unknown) & $0 / 12 / 1$ & $4 / 9 / 2$ \\
\hline
\end{tabular}

Definition of abbreviations: reversibility of $\mathrm{FEV}_{1}$ after $400 \mu \mathrm{g}$ salbutamol, $\mathrm{dFEV}_{1}$; forced expiratory volume in $1 \mathrm{~s}, \mathrm{FEV}_{1}$; forced vital capacity, FVC; carbon monoxide diffusion constant, Kco; residual volume, RV.

* $P<0.005$ versus subjects without COPD.

${ }^{\dagger} \mathrm{FEV}_{1} / \mathrm{FVC}$ is given as actual ratio in $\%$.

of staining obtained with a series of dilutions, which produced specific and easily visible signals on paraffin sections made from the same control tissue before performing the staining protocol on all sections. To avoid day-to-day variations in staining intensities, the incubations of all specimens with each antibody were performed in one single run. Sections were deparaffinized and rehydrated before incubation with specific mouse monoclonal and affinity-purified antibodies against FGF-1 (1:2000 dilution), FGF-2 (1:200 dilution) and FGFR-1 (1:2000 dilution). The mouse IgG1 antibody against human FGF-1 was raised using a synthetic peptide corresponding to the internal 61-99 amino acid sequence, whereas the mouse IgG2b antibody was raised against a synthetic peptide corresponding to the 16 amino acids from the C-terminus of human FGFR-1, as described previously $(17,18)$. FGF-2 was a mouse (IgG1 isotype) monoclonal antibody raised against human FGF-2 (molecular weight: $18-24 \mathrm{kD}$ ), which was procured from Transduction Laboratories (Lexington, KY). Antihuman mouse monoclonal antibodies against $\alpha$-smooth muscle actin ( $\alpha$-SMA), Ki-67 and FGF-2 were purchased from NeoMarkers (Clone 1A4; Fremont, CA), from Biogenex (San Ramon, CA) and from Transduction Laboratories, respectively. To block nonspecific binding, sections were preincubated with $10 \%$ normal goat serum diluted in $5 \%$ BSA in PBS (pH 7.4). Subsequently, sections were incubated overnight at $4^{\circ} \mathrm{C}$ with the primary antibodies (FGF-1 and FGFR-1) diluted appropriately, or for $1 \mathrm{~h}$ at room temperature in the case of $\alpha$-SMA (1:1000 dilution). Secondary biotinylated anti-immunoglobulins (Multilink, 1:75 dilution; Biogenex) and tertiary streptavidin conjugated alkaline phosphatase (Label 1:50 dilution; Biogenex) were used to enhance the detection sensitivity. Color was developed using new fuchsin, while endogenous alkaline phosphatase activity was inhibited by $0.01 \mathrm{M}$ levamisole.

FGF-2 and Ki-67 immunostaining was performed on serial sections after antigen retrieval by boiling in $10 \mathrm{mM}$ citrate buffer $(\mathrm{pH}$ 6.0) for $10 \mathrm{~min}$ in a microwave oven. Sections were preincubated with $10 \%$ normal goat serum in 5\% BSA/PBS, followed by incubation with primary antibody (1:50 dilution) overnight at $4^{\circ} \mathrm{C}$. Slides were rinsed in PBS, incubated for $30 \mathrm{~min}$ with peroxidase conjugated streptavidin at a dilution of 1:50 (Biogenex). Subsequently, sections were colored using 0.025\% 3,3-diaminobenzidine (Sigma, St. Louis, MO) in 0.01 molar PBS, containing $0.03 \%$ hydrogen peroxide. Slides were counterstained with Mayer's hematoxylin. Positive controls consisted of human breast carcinoma and placen- 

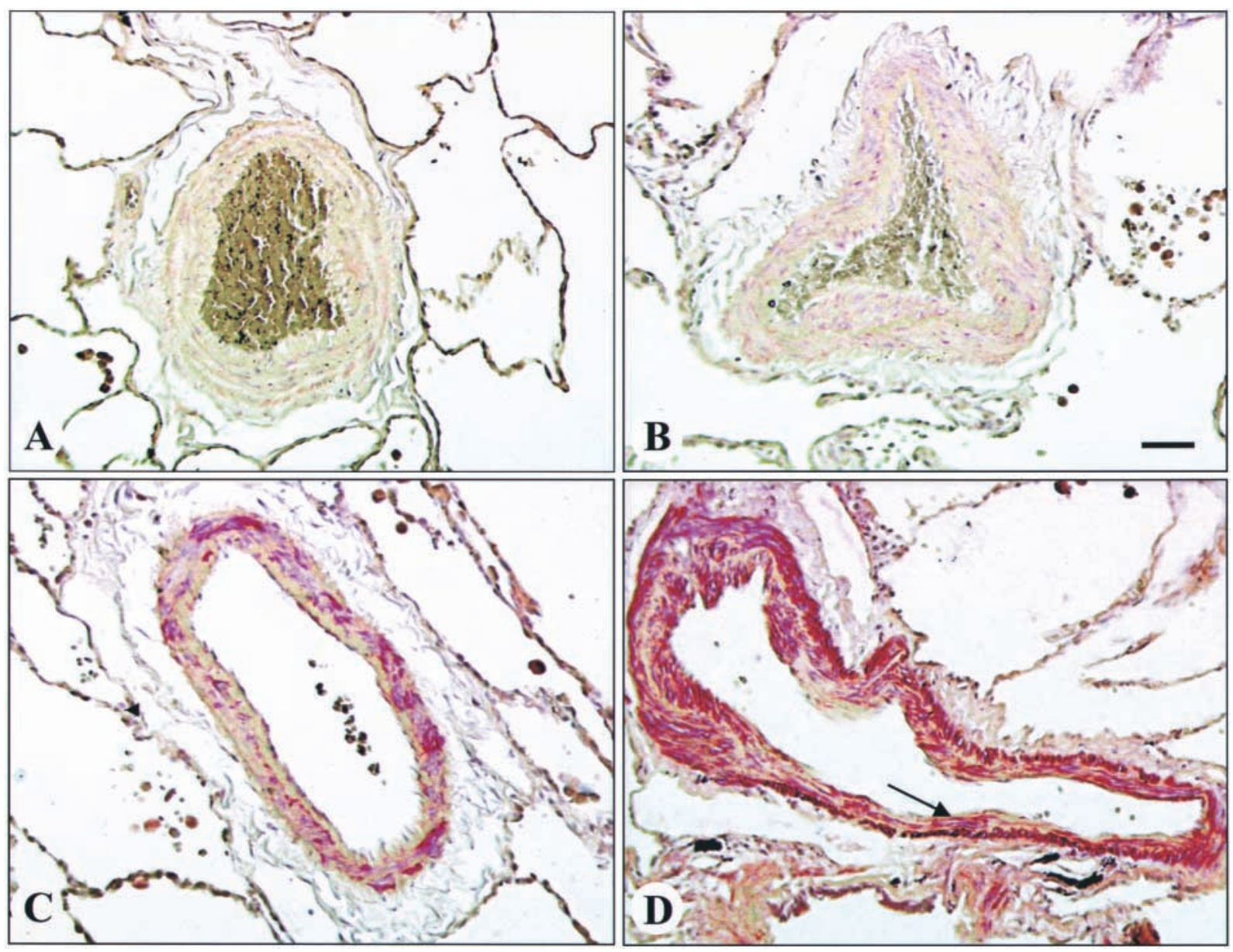

Figure 1. Representative examples of staining intensity pattern used for visual scoring Photomicrographs depict lung tissue sections from patients without $\operatorname{COPD}(A$ and $C)$ and with COPD $(B$ and $D)$, showing FGFR-1 staining (red new-fuchsine) in vascular smooth muscle cells. $A$ to $D$ show representative examples of staining intensities used for visual scoring, $0-3$ respectively. Scale bar $=50 \mu \mathrm{m}$; original magnification: $\times 100$.

tal tissue. The optimal dilutions for all antibodies were identified by examining the intensity of staining obtained with a series of dilutions, which produced specific and easily visible signals on paraffin sections derived from the same control tissue. Slides were mounted and staining results were systematically investigated (see below). Negative controls consisted of omission of the primary antibody.

\section{Semiquantitative Analysis}

All tissues were analyzed in a blinded fashion in random order by two independent observers who were unaware of the clinical data of the case under study. Semiquantitative analysis was performed using an arbitrary visual scale with grading scores of $0,1,2$, and 3 representing no, weak, moderate and intense staining, respectively $(14,15)$. Errors within and between observers were assessed by correlating the expression scores using Pearson's analysis, which indicated a very high correlation, ranging from 0.8 to 0.9 . Microphotographs in Figures $1 \mathrm{~A}$ to $1 \mathrm{D}$ show representative examples of staining intensities used for visual scores $0-3$, respectively. Sections were graded for the intensity of signal expression of FGF-1, FGF-2, and FGFR-1 in the endothelium and VSM of small $(50-200 \mu \mathrm{m}$ inside diameter [ID]) and in the endothelium, VSM, and adventitial area of large $(>200 \mu \mathrm{m}$ ID) pulmonary arteries.

\section{Video Image Analysis}

In addition, video image analysis was performed for $\alpha$-SMA staining using Leica Qwin system version 3.0 (Leica B.V., Rijswijk, The Netherlands). Twenty digital images $(736 \times 574$ pixels $)$ from each section were taken using a video camera. ID of blood vessels was derived as a mean of measured vertical and horizontal diameters. In our study, we excluded those vessels with a vertical to horizontal ID ratio $>3$. Based on the ID, pulmonary vessels were grouped into four sizes $(50-100 \mu \mathrm{m}, 100-200 \mu \mathrm{m}, 200-400 \mu \mathrm{m}$ and $>400$ $\mu \mathrm{m})$. VW area, $\alpha$-SMA stained area and vessel ID were measured. Measurements were expressed as percentages of staining per VW $\left(\alpha-\mathrm{SMA} / \mathrm{VW}_{\text {area }}\right)$, for $\mathrm{VW}_{\text {area }}$ corrected for ID ( $\left.\mathrm{VW}_{\text {area }} / \mathrm{ID}\right)$ and $\alpha$-SMA staining, also corrected for lumen diameter $\left(\alpha-\mathrm{SMA}_{\text {area }} / \mathrm{ID}\right)$.

\section{Statistical Analysis}

Data were analyzed for statistical significance using the unpaired, two-tailed Students' $t$ test as well as the Mann-Whitney nonpara- 
metric test, when appropriate (14, 15, SPSS; SPSS Incorporated, Chicago, IL). The staining score data for FGF-1, FGF-2 and FGFR-1 were expressed as mean \pm SEM. Furthermore, FGF-1, FGF-2, and FGFR-1 staining scores for different vessels with ID > $200 \mu \mathrm{m}$ and for those $<200 \mu \mathrm{m}$ were correlated with $\mathrm{FEV}_{1}$ using Pearson's correlation analysis. Furthermore, the individual FEV values were correlated with the vascular remodeling data $\left(\mathrm{VW}_{\text {area }}\right)$ ID) in both the groups. Differences with $P \leqslant 0.05$ were considered to be statistically significant.

\section{Results}

\section{Clinical Parameters}

The clinical and lung function characteristics of all subjects included in the study are listed in Table $1 . \mathrm{FEV}_{1}$ and $\mathrm{FEV}_{1} /$ FVC values were significantly lower in the group with COPD than in the group without COPD $(P<0.001)$. In the group with COPD, residual volume was increased, whereas $\mathrm{K}_{\mathrm{co}}$ was reduced $(P<0.005)$. The subjects in the two groups did not differ significantly with respect to age, total lung capacity, reversibility in $\mathrm{FEV}_{1}$, smoking status (pack-years), or previous steroid usage (Table 1).

\section{Localization and Quantification of FGF-1 and FGF-2}

FGF-1 expression was detected in the media and adventitia of large pulmonary arteries and veins, but only in the media of small vessels. No FGF-1 staining was found in the endothelial layer of any vessels. FGF-2 was localized specifically in nuclei of endothelial and vascular smooth muscle cells. Expression of FGF-1 and FGF-2 was also observed in epithelial and bronchiolar smooth muscle cells. Representative microphotographs showing the expression patterns of FGF-1 and FGF-2 are presented in Figure 2. A summary of the semiquantitative data of FGF-1 and FGF-2 immunostaining is presented in Figure 3. In subjects with COPD, we observed significantly increased $(P<0.02)$ expression of FGF-1 in medial VSM of larger vessels, but the level of adventitial expression of FGF-1 remained unaltered (Figure $3 \mathrm{~A}$ ). In contrast to FGF-1, the expression of FGF-2 was elevated in the group with COPD, but only in the small vessels with an ID $<200 \mu \mathrm{m}$, where it was increased in endothelial $(P<0.007)$ and medial smooth muscle $(P<$ 0.001 ) cells (Figure 3B).
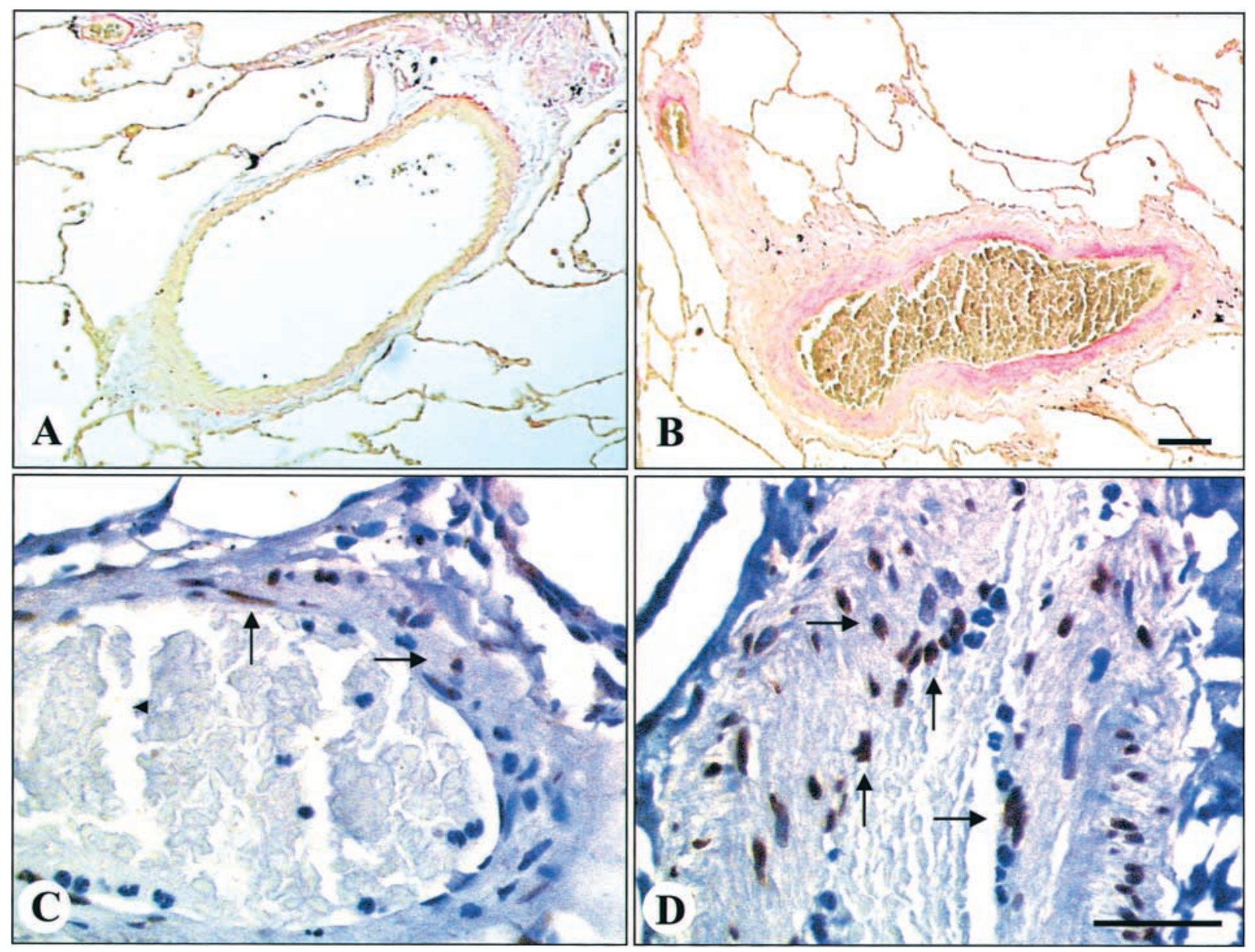

Figure 2. Photomicrographs of lung tissue sections from patients without COPD $(A$ and $C$ ) and with COPD $(B$ and $D)$. $A$ and $B$ (scale bar $=50 \mu \mathrm{m}$; original magnification: $\times 100)$ show representative examples of FGF-1 protein staining (red new-fuchsine) in vascular smooth muscle cells of a large vessel (ID $>200 \mu \mathrm{m})$. $C$ and $D($ scale bar $=100 \mu \mathrm{m}$; original magnification: $\times 400)$ show representative examples of nuclear FGF-2 expression (brown 3,3-diaminobenzidine) in endothelium and vascular smooth muscle cells of vessels with ID $<200 \mu \mathrm{m}$. Arrows indicate positive nuclei. 

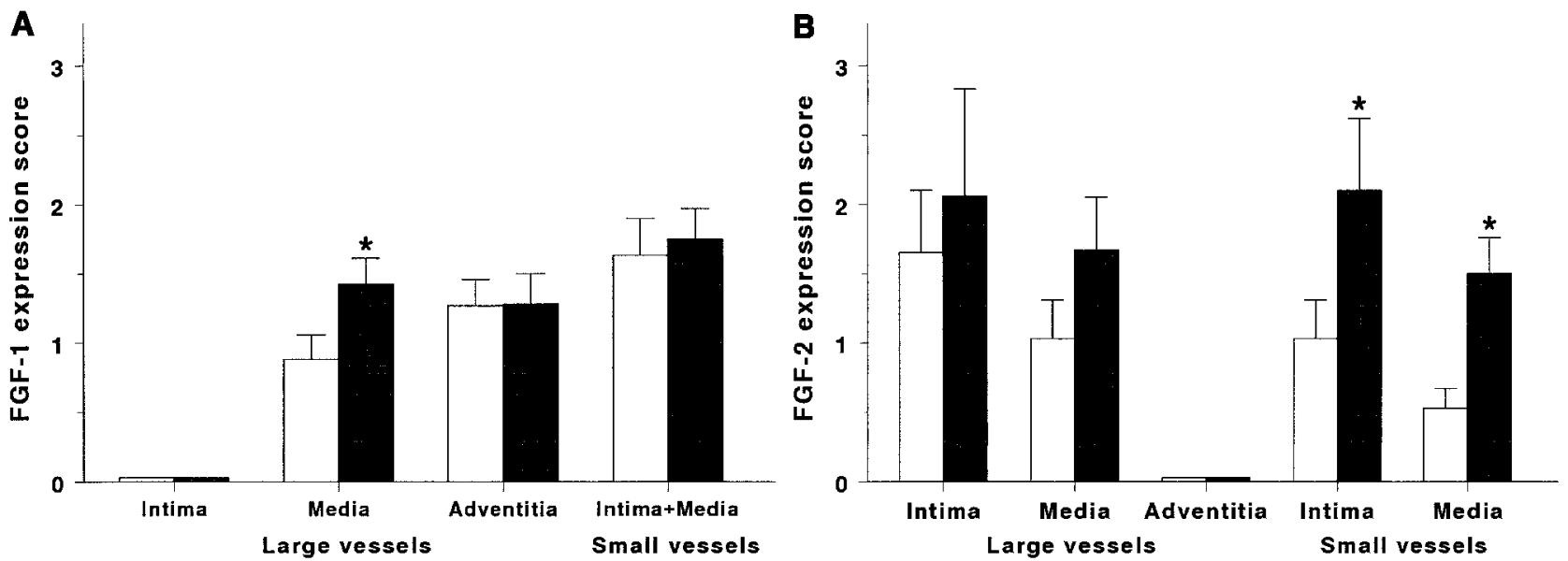

Figure 3. Graphic representations of FGF-1 $(A)$ and FGF-2 $(B)$ expression scores (mean \pm SEM) in large (ID $>200 \mu m)$ and small $(\mathrm{ID}<200 \mu \mathrm{m})$ vessels in groups without COPD (open bars) and with COPD (closed bars). * $P<0.05$ versus the group without COPD.

\section{Localization and Quantification of FGFR-1}

FGFR-1 immunoreactivity was detected in epithelial and bronchiolar smooth muscle cells, and in the endothelium and vascular smooth muscle of large and small vessels. No adventitial positivity for FGFR-1 was observed. Representative microphotographs showing the expression pattern of FGFR-1 are presented in Figure 4. A graphic representation of the data of FGFR-1 immunostaining is displayed in Figure 5. The expression of FGFR-1 was significantly elevated in medial smooth muscle cells of large vessels $(P<$ 0.04) in the group with COPD as compared with the group without COPD, whereas the staining for the receptor in the intimal endothelium remained unaltered. Moreover, in contrast to the FGF-1 expression in small vessels in patients with COPD, we found significantly higher expression levels of the receptor $(P<0.05)$ in medial smooth muscle of small vessels (Figure 5).

\section{Assessment of Vascular Remodeling}

To examine pulmonary vascular remodeling as evidenced in variations in wall thickness and muscular medial thickness, video image analysis was performed using $\alpha$-SMA immunostaining. Four separate groups with vessels of 50-100, 100200, 200-400 and $>400 \mu \mathrm{m}$ ID, respectively, were analyzed (Figure 6). Measurements (mean \pm SEM) were expressed as $\mathrm{VW}_{\text {area }} / \mathrm{ID}, \alpha-\mathrm{SMA}_{\text {area }} / \mathrm{ID}$, or percentage $\alpha$-SMA staining per $\mathrm{VW}_{\text {area }}$ that represents the volume fraction for smooth muscle staining $\left(\alpha-\mathrm{SMA} / \mathrm{VW}_{\text {area }}\right)$. The graphic representation of VW is presented in Figure 7. A significant increase was seen in $\mathrm{VW}_{\text {area }}$ /ID ratio for COPD in vessels of 100-200 $\mu \mathrm{m}(44.2 \pm 1.9$ versus $36.4 \pm 2.1, P=0.007), 200-400 \mu \mathrm{m}$ $(57.9 \pm 2.4$ versus $44.7 \pm 3.2, P<0.001)$ and $>400 \mu \mathrm{m}$ $(75.6 \pm 2.6$ versus $56.8 \pm 5.9, P=0.011)$ (Figure 7A). In vessels ranging from 50 to $100 \mu \mathrm{m}$ in ID, no differences in $\mathrm{VW}_{\text {area }} / \mathrm{ID}$ ratio were observed. A significant increase in $\alpha-\mathrm{SMA}_{\text {area }} / \mathrm{ID}$ ratio was observed for COPD in the $200-400$ $\mu \mathrm{m}$ ID $(26.7 \pm 1.9$ versus $20.3 \pm 2.9, P=0.034)$ and $>400$ $\mu \mathrm{m}$ ID $(38.3 \pm 2.0$ versus $23.5 \pm 3.4, P=0.006)$ vessels, but not in the 50-100 $\mu \mathrm{m}$ and 100-200 $\mu \mathrm{m}$ vessels. Surpris- ingly, no significant differences were observed between the groups with and without COPD in the percent of vascular smooth muscle, defined as $\alpha-\mathrm{SMA} / \mathrm{VW}_{\text {area }}$ in any vessel type (Figure 7B). Proliferation of VSM cells, as evidenced from Ki-67 positivity, was observed infrequently (data not shown).

\section{Correlation with Clinical Data}

The staining scores of FGF-1, FGF-2, and FGFR-1 expression in patients with COPD and those without COPD were analyzed using Pearson's test. For FGF-1, we observed a weak but significant inverse correlation $(r=-0.39, P=$ 0.038 ) between staining score and $\mathrm{FEV}_{1}$ in the medial VSM of vessels $>200 \mu \mathrm{m}$ in ID (Figure 8A). In addition, there was a significant inverse correlation of FGF-2 staining scores in both endothelium $(r=-0.44, P=0.002)$ and medial VSM $(r=-0.55, P<0.0001)$ of vessels $<200 \mu \mathrm{m}$ in ID with $\mathrm{FEV}_{1}$ (Figures 8B and 8C). However, in vessels $>200$ $\mu \mathrm{m}$ in ID, no significant correlation between FGF-2 expression and $\mathrm{FEV}_{1}$ was found (data not shown). Surprisingly, staining scores for FGFR-1 were not significantly correlated with $\mathrm{FEV}_{1}$ (data not shown). When considering the association between $\mathrm{FEV}_{1}$ and medial hypertrophy ( $\mathrm{VW}_{\text {area }} / \mathrm{ID}$ ratio), we observed a significant inverse correlation of -0.42 $(P=0.007)$ for vessels $>200 \mu \mathrm{m}$ ID (Figure 8D). However, no significant correlation could be established between $\mathrm{FEV}_{1}$ and $\mathrm{VW}_{\text {area }} / \mathrm{ID}$ ratio for the vessels $<200 \mu \mathrm{m}$ ID $(r=$ $-0.10, P>0.10)$.

\section{Discussion}

In this study we have found that COPD is associated with an increase in the expression of FGF-2 in small pulmonary vessels $(<200 \mu \mathrm{m})$ and FGF-1 in large $(>200 \mu \mathrm{m})$ pulmonary vessels, whereas FGFR-1 is increased in both vessel types. Vascular medial thickness, assessed by video image analysis, was significantly increased in patients with COPD in pulmonary vessels of various sizes. Pearson's correlation analysis revealed a significant inverse correlation of $\mathrm{FEV}_{1}$ with FGF-1 staining in the media of large vessels and with 

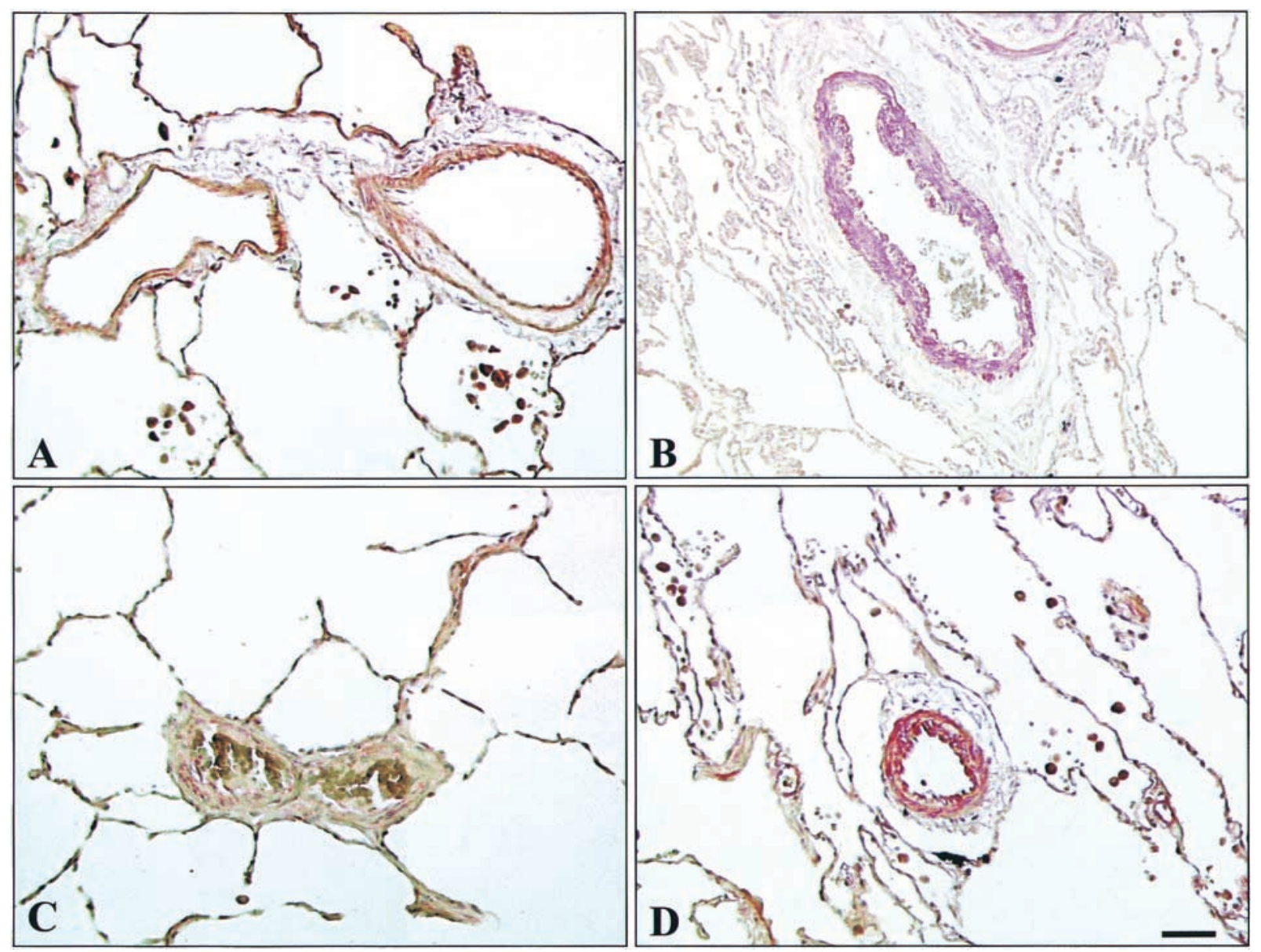

Figure 4. Photomicrographs of lung tissue sections from patients without COPD $(A$ and $C$ ) and with COPD $(B$ and $D)$ showing FGFR-1 staining (red new-fuchsine) in vascular smooth muscle cells from large (ID $>200 \mu \mathrm{m} ; A$ and $B$ ) and small (ID $<200 \mu \mathrm{m} ; C$ and $D$ ) blood vessels. Scale bar $=50 \mu \mathrm{m}$; original magnification: $\times 100$.

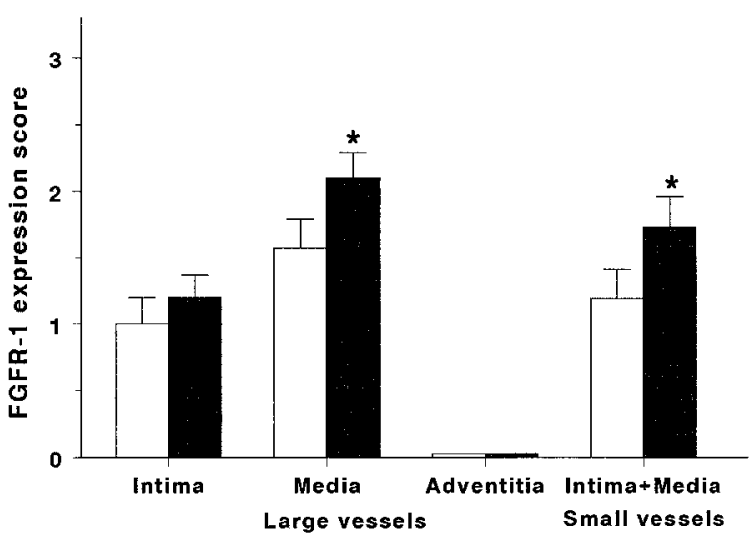

Figure 5. Graphic representation of FGFR-1 expression scores $($ mean \pm SEM) in large (ID $>200 \mu \mathrm{m})$ and small (ID $<200 \mu \mathrm{m})$ vessels in groups without COPD (open bars) and with COPD (closed bars). $* P<0.05$ versus the group without COPD.
FGF-2 expression in both endothelium and VSM of small vessels. In addition, an inverse correlation of $\mathrm{FEV}_{1}$ with medial thickening was found in pulmonary vessels of larger caliber, indicating that the degree of pulmonary vascular remodeling is related to the severity of obstructive lung function defect.

Several studies have commented on the importance of structural and functional abnormalities in the pulmonary vasculature of patients with COPD. Hypoxia is known to induce prompt and severe vasoconstriction in the pulmonary vasculature, and sustained lung tissue hypoxia, as results from obstructive lung disease such as COPD, leads to pulmonary hypertension $(4,19)$. Hypoxic vasoconstriction is considered to represent one of the major contributing factors of pulmonary hypertension and right-sided heart failure in COPD and other chronic pulmonary diseases $(4$, 19). In addition, emphysema, accompanied by loss of elastic recoil, increased pulmonary pressure, and destruction of part of the pulmonary microvasculature may contribute to the increased vascular resistance observed in $\operatorname{COPD}(5,6)$.

Using video image analysis, we assessed systematically VW thickening in patients with COPD and non-COPD 

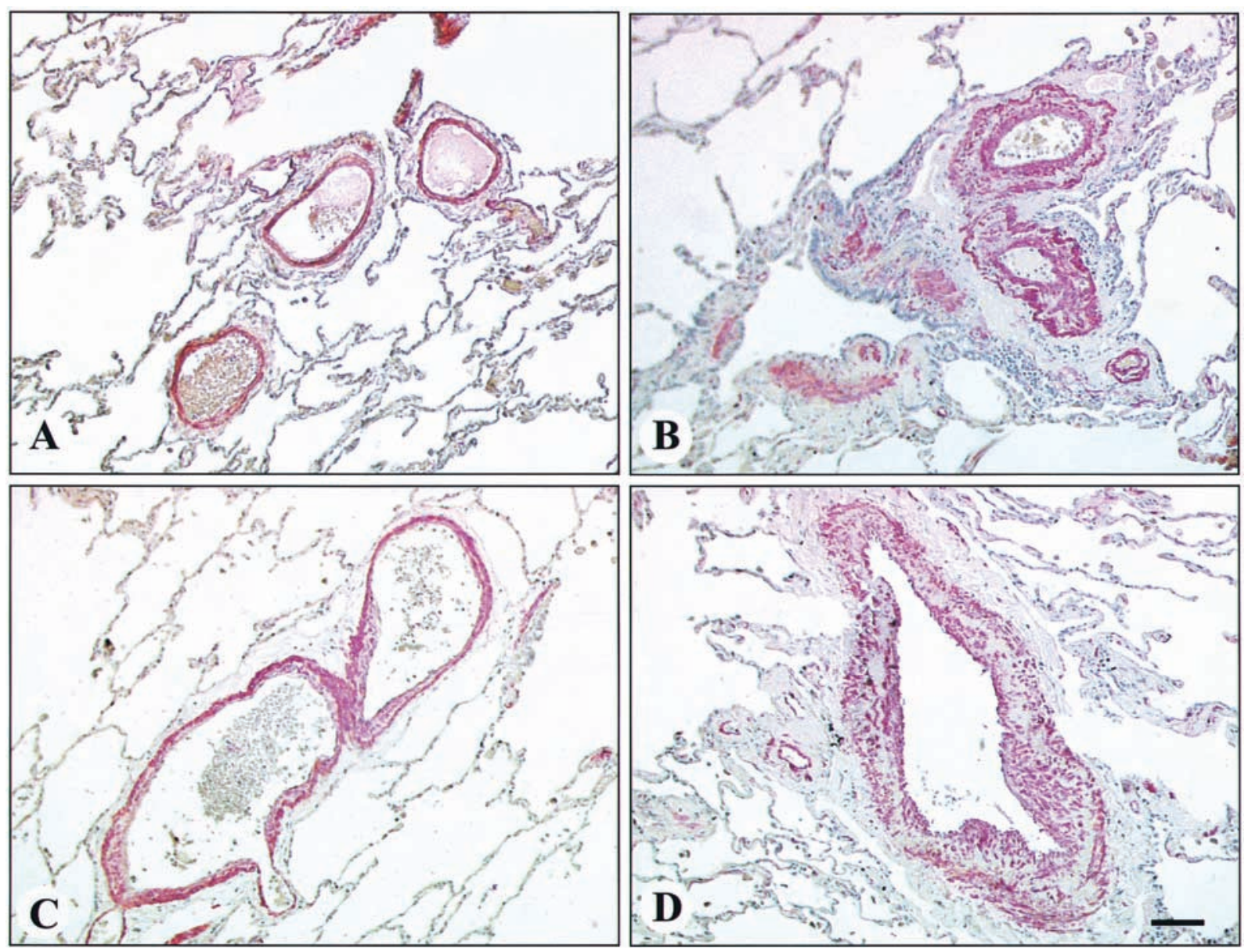

Figure 6. Photomicrographs of lung tissue sections from patients without COPD $(A$ and $C)$ and with COPD $(B$ and $D)$ showing $\alpha$-smooth muscle actin staining (red new-fuchsine) in vascular smooth muscle cells from small (ID $<200 \mu \mathrm{m} ; A$ and $B$ ) and large (ID $>200 \mu \mathrm{m}$; $C$ and $D$ ) blood vessels. Scale bar $=50 \mu \mathrm{m}$; original magnification: $\times 100)$.

cases. Wall thickness of vessels $200 \mu \mathrm{m}$ or more in diameter was increased in patients with COPD. Our results on pulmonary vascular remodeling, particularly in terms of intimal and medial thickening, are in agreement with several earlier reports $(4,5,20-22)$. Furthermore, the degree of intimal and medial thickening was correlated with the decrease in lung function and, hence, with the severity of the disease.
Wright and coworkers $(5,23)$ also observed a correlation with the severity of disease in patients with mild to moderate COPD with intimal thickening and in those with severe COPD with medial thickening. Similar findings on vascular abnormalities in patients with COPD were recently reported by Peinado and coworkers, who showed intimal but not medial thickening in the vasculature of patients with
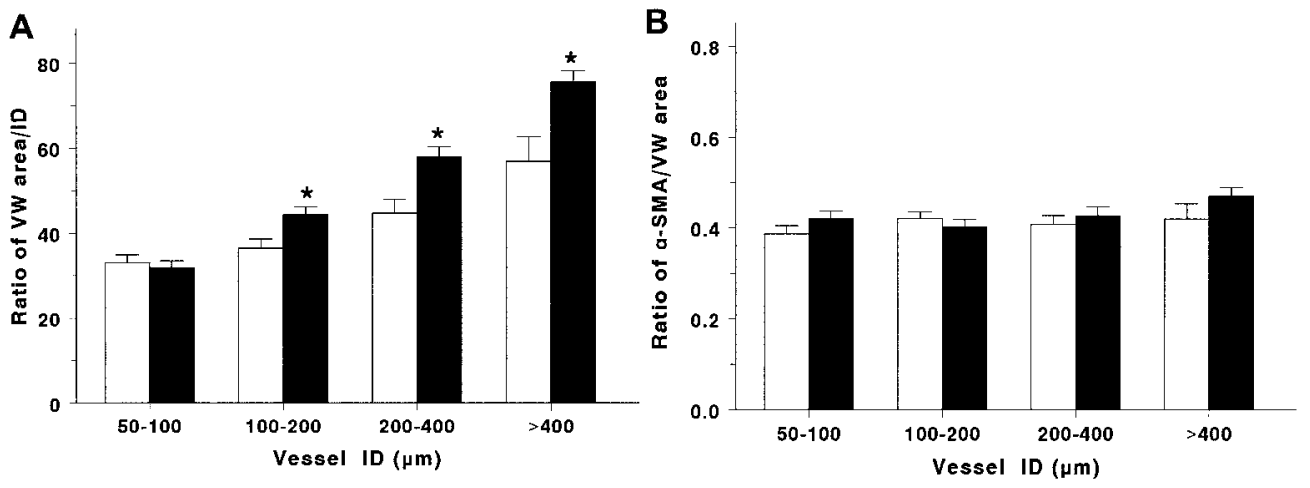

Figure 7. Graphic representations of VW measurements (mean \pm SEM) using video image analysis in the group without COPD (open bars) and that with COPD (closed bars). (A) Ratio of vascular wall area/ID ( $\left.\mathrm{VW}_{\text {area }} / \mathrm{ID}\right)$ ratio. (B) Ratio of $\alpha-\mathrm{SMA}_{\text {area }} /$ $\mathrm{VW}_{\text {area. }} * P<0.05$ versus the group without COPD. 
A
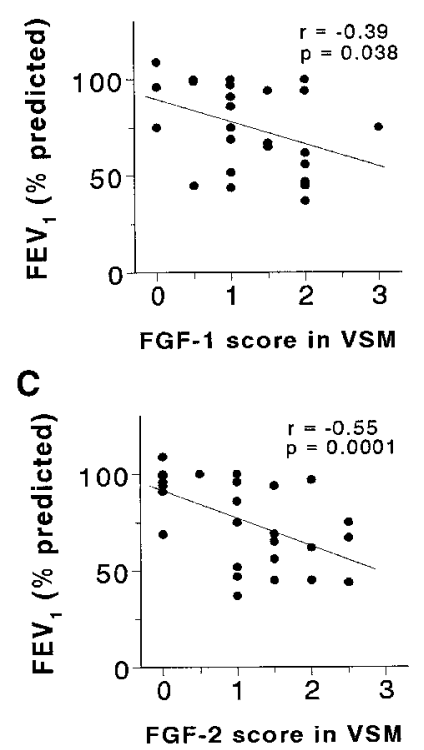

B

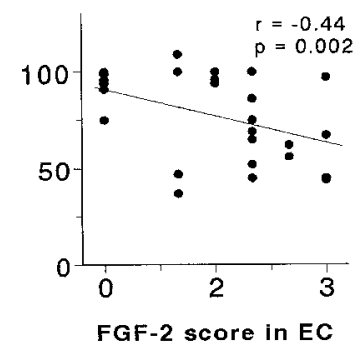

D

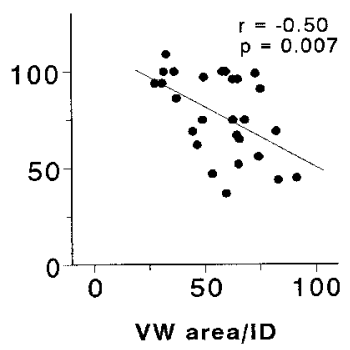

Figure 8. Correlation with $\mathrm{FEV}_{1} \%$ predicted of $(A) \mathrm{FGF}-1$ expression in vascular smooth muscle cells $(V S M$; ID $>200 \mu \mathrm{m}),(B)$ FGF-2 expression in endothelial cells $(E C)$ from small blood vessels (ID $<200 \mu \mathrm{m}),(C)$ FGF-2 expression in $V S M$ cells (ID < $200 \mu \mathrm{m})$, and $(D)$ vascular wall area/ID $\left(V W_{\text {ared }} / I D\right)$. Correlation coefficient $(r)$ and significance level ( $P$ value) were obtained using Pearson's linear regression analysis.

mild COPD compared with nonsmoking control subjects $(20,21)$.

We used expression of smooth muscle marker $\alpha$-SMA (24) to investigate whether the $\alpha-\mathrm{SMA} / \mathrm{VW}_{\text {area }}$ ratio of smooth muscle in the VW had changed during the progression of COPD. Surprisingly, the ratio remained unchanged. Approximately $42 \%$ of cells in all vessels stained positive for $\alpha$-SMA, indicating that the increase in wall thickness could be attributed to the deposition of extracellular matrix proteins and the medial accumulation of other cells, such as inflammatory cells and fibroblasts. Recently, we found specific staining for extracellular matrix proteins, like fibronectin and collagen subtypes, in the intimal vascular cells of these pulmonary vessels, indicating ongoing intimal fibrosis in patients with COPD (data not shown). Taken together, the data from this study indicate that vascular remodeling in patients with COPD could be a contributing event in the pathogenesis of pulmonary hypertension in these patients. Furthermore, the observed changes in the intimal fibrosis as well as medial thickening could narrow the vessel caliber and may eventually lead to more severe vascular obstruction in patients with COPD.

Members of the FGF family FGF-1, FGF-2, and FGFR-1 are constitutively expressed in normal human lungs, particularly in airway epithelium and monocytes, and are localized in the intima and media of pulmonary vessels (25). Pulmonary expression patterns of FGF-1, FGF-2, and FGFR-1, as found in our study, are in agreement with results obtained by Hughes and Hall (25) in normal lungs. However, in the

peripheral regions of the lungs of patients with COPD, we also observed FGF-1 in adventitia and FGF-2 immunoreactivity in the nuclei of medial smooth muscle and endothelial cells, suggesting a potential role of the FGF-FGFR system in vascular remodeling in patients with COPD.

FGF family members are implicated in tissue remodeling in a wide variety of pathophysiologic conditions, including systemic hypertension, ischemic heart disease, and interstitial lung fibrosis $(10,12,26,27)$. Barrios and coworkers (10) showed FGF-1 and FGFR-1 expression in experimentally induced pulmonary fibrosis. Becerril and colleagues found that FGF-1 expression in the lung fibroblasts results in downregulation of collagen synthesis and upregulation of collagenases, which may protect against fibrosis (28). However, increased FGF-2 and FGFR-1 expression in vascular smooth muscle cells in vitro in response to vascular injury has been shown to be associated with extracellular matrix remodeling, cellular proliferation, downregulation of collagen type I, and upregulation of collagenase MMP-1 (29). Our findings of upregulated FGF-1, FGF-2, and FGFR-1 expression could indicate that such compensatory mechanisms are active in COPD because smoking has been suggested to affect cellular viability in lungs.

In a recent study, Singh and colleagues demonstrated that increased nuclear expression of FGF-2 in vascular smooth muscle and endothelium precedes arterial enlargement in response to increased arterial blood flow in vivo (12). Though the function of high molecular weight FGF-2 in the cell nucleus remains unclear, it is believed that this form of FGF-2 translocates to the nucleus. Moreover, Stachowiak and coworkers have demonstrated colocalization of FGFR-1 and FGF-2 in the nucleus of human astrocytes, suggesting novel mechanisms for the action of FGF-2 (30). In this study, we show that FGF-2 is localized in the nucleus of endothelial and VSM cells and that its expression is increased in pulmonary vessels with diameter $>200 \mu \mathrm{m}$ in patients with COPD, indicating a role for this growth factor in vascular remodeling. We also show that in patients with COPD, the expression of FGF-2 was upregulated in vessels with an ID of $>200 \mu \mathrm{m}$. Bryant and coworkers (13) recently found that administration of FGF-2 could inhibit internal luminal area decrease and wall thickening in response to altered blood flow; furthermore, this inhibitory effect could be blocked by anti-FGF-2 neutralizing antibodies. Our findings suggest that FGF-2 plays an important role in the response to increased pressure in the pulmonary vasculature of patients with COPD. Several studies on hypoxia-induced pulmonary hypertension have shown that increased smooth muscle mass develops as a result of hypertrophy and hyperplasia of pre-existing smooth muscle cells, or results from differentiation of fibroblasts recruited to the media from the adventitia $(24,31)$. It is likely that such mechanisms of vascular smooth muscle mass increase are operational in COPD-related vascular remodeling.

In vivo and in vitro data indicate that smooth muscle cells, and their cross-talk with the endothelium, myofibroblasts, and inflammatory cells via growth factors and cytokines, are major contributing factors to vascular remodeling during different pathophysiologic conditions $(24,28$, $32,33)$. Furthermore, inflammation, a well-established fac- 
tor in peripheral as well as in central airways in patients with COPD, could also be associated with vascular remodeling in patients with COPD. Increased adventitial infiltration of inflammatory cells, predominantly $\mathrm{CD}^{+} \mathrm{T}$ lymphocytes in muscular pulmonary and bronchiolar arteries, has been reported $(21,34)$. Taken together, our results support the notion that in patients with COPD, increased vascular expression of FGF-1, FGF-2, and FGFR-1 could participate in an autocrine and/or a complex growth factor-cytokine interactive manner in regulating the process of pulmonary vascular remodeling. Our data further support the hypothesis that COPD is associated with pulmonary vascular remodeling and that the FGF-FGFR system contributes to the pathogenesis and severity of the disease.

Acknowledgments: The authors thank Drs. J. Stolk (Leiden University Medical Center) and R. Slingerland (Southern Hospital, Rotterdam) for their help in the analysis of the clinical data. The expert technical assistance of Mrs. E. Yilmaz and Mrs. A. Willems-Widyastuti is also acknowledged. This study was supported by The Netherlands Asthma Foundation (grant \#97.73).

\section{References}

1. Barnes, P. J. 1998. Chronic obstructive pulmonary disease: new opportunities for drug development. Trends Pharmacol. Sci. 19:415-423.

2. Madison, J. M., and R. S. Irwin. 1998. Chronic obstructive pulmonary disease. Lancet 352:467-473.

3. Barnes, P. J. 1998. Pharmacology of airway smooth muscle. Am. J. Respir. Crit. Care Med. 158:S123-132.

4. Wright, J. L., L. Lawson, P. D. Pare, R. O. Hooper, D. I. Peretz, J. M. Nelems, M. Schulzer, and J. C. Hogg. 1983. The structure and function of the pulmonary vasculature in mild chronic obstructive pulmonary disease. The effect of oxygen and exercise. Am. Rev. Respir. Dis. 128:702-707.

5. Magee, F., J. L. Wright, B. R. Wiggs, P. D. Pare, and J. C. Hogg. 1988. Pulmonary vascular structure and function in chronic obstructive pulmonary disease. Thorax 43:183-189.

6. Jeffery, P. K. 1998. Structural and inflammatory changes in COPD: a comparison with asthma. Thorax 53:129-136.

7. Holgate, S. T. 1997. Asthma: a dynamic disease of inflammation and repair. Ciba Found. Symp. 206:5-34.

8. Werner, S. 1998. Keratinocyte growth factor: a unique player in epithelial repair processes. Cytokine Growth Factor Rev. 9:153-165.

9. Szebenyi, G., and J. F. Fallon. 1999. Fibroblast growth factors as multifunctional signaling factors. Int. Rev. Cytol. 185:45-106.

10. Barrios, R., A. Pardo, C. Ramos, M. Montano, R. Ramirez, and M. Selman. 1997. Upregulation of acidic fibroblast growth factor during development of experimental lung fibrosis. Am. J. Physiol. 273:L451-458.

11. al-Dossari, G. A., J. Jessurun, R. M. Bolman iii, V. R. Kshettry, M. B. King, J. J. Murray, and M. I. Hertz. 1995. Pathogenesis of obliterative bronchiolitis: possible roles of platelet-derived growth factor and basic fibroblast growth factor. Transplantation 59:143-145.

12. Singh, T. M., K. Y. Abe, T. Sasaki, Y. J. Zhuang, H. Masuda, and C. K. Zarins. 1998. Basic fibroblast growth factor expression precedes flowinduced arterial enlargement. J. Surg. Res. 77:165-173.

13. Bryant, S. R., R. J. Bjercke, D. A. Erichsen, A. Rege, and V. Lindner. 1999. Vascular remodeling in response to altered blood flow is mediated by fibroblast growth factor-2. Circ. Res. 84:323-328.

14. Grashoff, W. F., J. K. Sont, P. J. Sterk, P. S. Hiemstra, W. I. de Boer, J. Stolk, J. Han, and J. M. van Krieken. 1997. Chronic obstructive pulmonary disease: role of bronchiolar mast cells and macrophages. Am. J. Pathol. 151:1785-1790.

15. de Boer, W. I., A. van Schadewijk, J. K. Sont, H. S. Sharma, J. Stolk, P. S. Hiemstra, and J. H. van Krieken. 1998. Transforming growth factor $\beta 1$ and recruitment of macrophages and mast cells in airways in chronic obstructive pulmonary disease. Am. J. Respir. Crit. Care Med. 158:19511957.
16. Quanjer, P. H., G. J. Tammeling, J. E. Cotes, O. F. Pedersen, R. Peslin, and J. C. Yernault. 1993. Lung volumes and forced ventilatory flows: report working party standardization of lung function tests, European Community for Steel and Coal. Official statement of the European Respiratory Society. Eur. Respir. J. Suppl. 16:5-40.

17. Coope, R. C., P. J. Browne, C. Yiangou, G. S. Bansal, J. Walters, N. Groome, S. Shousha, C. L. Johnston, R. C. Coombes, and J. J. Gomm. 1997. The location of acidic fibroblast growth factor in the breast is dependent on the activity of proteases present in breast cancer tissue. Br. J. Cancer 75:1621-1630.

18. Yiangou, C., H. Cox, G. S. Bansal, R. Coope, J. J. Gomm, R. Barnard, J. Walters, N. Groome, S. Shousha, R. C. Coombes, and C. L. Johnston. 1997. Down-regulation of a novel form of fibroblast growth factor receptor 1 in human breast cancer. Br. J. Cancer 76:1419-1427.

19. Wright, J. L., T. Petty, and W. M. Thurlbeck. 1992. Analysis of the structure of the muscular pulmonary arteries in patients with pulmonary hypertension and COPD: National Institutes of Health nocturnal oxygen therapy trial. Lung 170:109-124.

20. Peinado, V. I., J. A. Barbera, J. Ramirez, F. P. Gomez, J. Roca, L. Jover, J. M. Gimferrer, and R. Rodriguez-Roisin. 1998. Endothelial dysfunction in pulmonary arteries of patients with mild COPD. Am. J. Physiol. 274: L908-913.

21. Peinado, V. I., J. A. Barbera, P. Abate, J. Ramirez, J. Roca, S. Santos, and R. Rodriguez-Roisin. 1999. Inflammatory reaction in pulmonary muscular arteries of patients with mild chronic obstructive pulmonary disease. Am. J. Respir. Crit. Care Med. 159:1605-1611.

22. Barbera, J. A., A. Riverola, J. Roca, J. Ramirez, P. D. Wagner, D. Ros, B. R. Wiggs, and R. Rodriguez-Roisin. 1994. Pulmonary vascular abnormalities and ventilation-perfusion relationships in mild chronic obstructive pulmonary disease. Am. J. Respir. Crit. Care Med. 149:423-429.

23. Wright, J. L., L. M. Lawson, P. D. Pare, B. J. Wiggs, S. Kennedy, and J. C. Hogg. 1983. Morphology of peripheral airways in current smokers and ex-smokers. Am. Rev. Respir. Dis. 127:474-477.

24. Jones, R., M. Jacobson, and W. Steudel. 1999. $\alpha$-smooth-muscle actin and microvascular precursor smooth-muscle cells in pulmonary hypertension. Am. J. Respir. Cell Mol. Biol. 20:582-594.

25. Hughes, S. E., and P. A. Hall. 1993. Immunolocalization of fibroblast growth factor receptor 1 and its ligands in human tissues. Lab. Invest. 69:173-182.

26. Liebler, J. M., M. A. Picou, Z. Qu, M. R. Powers, and J. T. Rosenbaum. 1997. Altered immunohistochemical localization of basic fibroblas growth factor after bleomycin-induced lung injury. Growth Factors 14: $25-38$.

27. Scheinowitz, M., D. Abramov, and M. Eldar. 1997. The role of insulin-like and basic fibroblast growth factors on ischemic and infarcted myocardium: a mini review. Int. J. Cardiol. 59:1-5.

28. Becerril, C., A. Pardo, M. Montano, C. Ramos, R. Ramirez, and M. Selman. 1999. Acidic fibroblast growth factor induces an antifibrogenic phenotype in human lung fibroblasts. Am. J. Respir. Cell Mol. Biol. 20:1020-1027.

29. Pickering, J. G., C. M. Ford, B. Tang, and L. H. Chow. 1997. Coordinated effects of fibroblast growth factor-2 on expression of fibrillar collagens, matrix metalloproteinases, and tissue inhibitors of matrix metalloproteinases by human vascular smooth muscle cells: evidence for repressed collagen production and activated degradative capacity. Arterioscler Thromb. Vasc. Biol. 17:475-482.

30. Stachowiak, M. K., P. A. Maher, A. Joy, E. Mordechai, and E. K. Stachowiak. 1996. Nuclear localization of functional FGF receptor 1 in human astrocytes suggests a novel mechanism for growth factor action. Brain Res. Mol. Brain Res. 38:161-165.

31. Jones, R., W. Steudel, S. White, M. Jacobson, and R. Low. 1999. Microvessel precursor smooth muscle cells express head-inserted smooth muscle myosin heavy chain (SM-B) isoform in hyperoxic pulmonary hypertension. Cell Tissue Res. 295:453-465.

32. Chen, C. H., and P. D. Henry. 1997. Atherosclerosis as a microvascular disease: impaired angiogenesis mediated by suppressed basic fibroblast growth factor expression. Proc. Assoc. Am. Physicians 109:351-361.

33. Ambalavanan, N., A. Bulger, and I. J. Philips. 1999. Hypoxia-induced release of peptide growth factors from neonatal porcine pulmonary artery smooth muscle cells. Biol. Neonate 76:311-319.

34. Saetta, M., A. Di Stefano, G. Turato, F. M. Facchini, L. Corbino, C. E. Mapp, P. Maestrelli, A. Ciaccia, and L. M. Fabbri. 1998. CD8+ T-lymphocytes in peripheral airways of smokers with chronic obstructive pulmonary disease. Am. J. Respir. Crit. Care Med. 157:822-826. 